\title{
Morbidité Des Enfants En Zones Urbaines Africaines. Le Cas De L'observatoire De Population De Ouagadougou (Burkina Faso)
}

\author{
Franklin Bouba Djourdebbé \\ Institut de Formation et de Recherche Démographiques (IFORD), \\ Université de Yaoundé II, Cameroun \\ Stéphanie Dos Santos \\ Laboratoire Population-Environnement-Développement-LPED \\ (IRD-AMU), Marseille, France \\ Institut Supérieur des Sciences de la Population (ISSP), \\ Université de Ouagadougou, Burkina Faso.
}

Thomas K. Legrand

Département de Démographie, Université de Montréal, Canada

Abdramane Bassiahi Soura

Institut Supérieur des Sciences de la Population (ISSP),

Université de Ouagadougou, Burkina Faso

Doi: 10.19044/esj.2018.v14n11p163 URL:http://dx.doi.org/10.19044/esj.2018.v14n11p163

\begin{abstract}
Rapid urbanization and its consequences in regard to access to water, sanitation, and waste management in African cities can be synonymous to health problems. Based on the data obtained from the Ouagadougou Health and Demographic Surveillance System, this paper focuses on characterizing most of those at risk of disease (fever, diarrhea, cough, infections of the skin and eyes). Spatial analysis show that populations in formal (zoned) neighbourhoods, compared to those in informal neighbourhoods, are most at risk of disease. However, in performing multiple correspondence factor analysis and classification, we found that the informal neighbourhoods are mostly at risk of disease. The formal and informal opposition is not absolute, but the differences remain strong despite the existence of atypical neighbourhoods. The contribution of the paper is to provide a new perspective for thinking in regards to the links between environment and children's health.
\end{abstract}

Keywords: Morbidity, children, environment, neighbourhoods, Burkina Faso 


\section{Résumé}

Les difficultés d'accès à l'eau et l'insalubrité dans les villes africaines sont parfois sources de maladies. En recourant aux analyses spatiales, factorielles et de classification, l'article décrit, à partir des données de l'Observatoire de population de Ouagadougou, les quartiers les plus à risque de morbidité : fièvre, diarrhée, toux, infections de la peau et des yeux. Les analyses spatiales basées sur la distance euclidienne montrent que les quartiers lotis sont les plus exposés aux dangers environnementaux. Pourtant, en effectuant des analyses factorielles et de classification, ce sont les quartiers non lotis qui courent plus de risques de maladies. Au-delà des différences loti/non loti, on constate la présence des quartiers atypiques dans tous les milieux de résidence. La dichotomie loti/non loti n'est pas tout à fait absolue du fait de l'hétérogénéité en termes de santé environnementale, notamment en zones non loties. Cet article fournit un nouvel éclairage sur les liens entre l'environnement et la santé chez les enfants.

Mots-clés : Morbidité, enfants, environnement, quartiers, Burkina-Faso

\section{Introduction}

Au cours des deux derniers siècles, la proportion de la population mondiale qui vit dans les villes est passée de 5 à $50 \%$ (UN, 2014). Les estimations des Nations Unies indiquent qu'en 2050, plus des deux tiers de la population mondiale vivront en ville (FNUAP, 2008). L'Afrique subsaharienne est la région du monde où l'augmentation de la population urbaine est la plus rapide (ONU-Habitat, 2010). La croissance annuelle de la population urbaine $(5 \%)$ y est deux fois supérieure à celle des pays d'Amérique latine ou d'Asie (UN, 2014). Selon les projections, l'Afrique subsaharienne devrait voir sa population urbaine multipliée par trois entre 2010 et 2050 (ONU-Habitat, 2010), ce qui constitue des défis majeurs pour les municipalités qui peinent à fournir un accès à l'eau potable et à l'assainissement (Hopewell \& Graham, 2014).

L'urbanisation est relativement récente en Afrique subsaharienne (Chen et al., 2014). Il s'agit d'un phénomène qui s'est produit essentiellement au cours des cinquante dernières années, quand la population urbaine est passée de 30 millions en 1950 à plus de 400 millions en 2010 (UN-Habitat, 2014). L'accroissement naturel de la population, la migration vers les villes, la sécheresse, ainsi que la pauvreté ayant entraîné le déplacement interne des populations ont accéléré le processus d'urbanisation en Afrique subsaharienne (Kimani-Murage et al., 2014). On estime qu'en 2025, l'Afrique subsaharienne comptera trois mégapoles, dont Lagos (18,9 millions d'habitants) et Kinshasa (14,5 millions) (UN-Habitat, 2014). L'insuffisance des infrastructures pour répondre à la croissance de la population urbaine a donné lieu au 
développement des quartiers informels (bidonvilles) (Hill et al., 2014). Dans les villes subsahariennes, les fosses septiques, les latrines mal construites et les zones inondées favorisent la contamination de l'eau, de même que la propagation de maladies liées à l'environnement comme le paludisme, les infections respiratoires aiguës et les maladies diarrhéiques (Walker et al., 2013; WHO \& UNICEF, 2014).

La mortalité et la morbidité des enfants restent élevées en milieu urbain en Afrique subsaharienne par rapport à d'autres régions du monde, même si elles ont connu une baisse observable ces dernières décennies (Fink et al., 2013; Kimani-Murage et al., 2014). Bien que 42\% de la population urbaine de l'Afrique subsaharienne ait accès à l'assainissement amélioré, son manque dans les quartiers informels en ville peut intensifier les risques de maladie, où vivent $62 \%$ des habitants des villes de l'Afrique subsaharienne aujourd'hui (WHO \& UNICEF, 2014). Même si la situation des enfants est en moyenne meilleure en milieu urbain par rapport au milieu rural, toutefois ces dernières années, suite à la crise économique, certaines sous-populations urbaines connaîtraient une mortalité et une morbidité supérieures à celles observées en milieu rural (Montgomery \& Hewett, 2005; Fink et al., 2014). Cependant, peu de travaux ont porté sur la morbidité environnementale en milieu urbain, même si des études récentes, basées sur les données des enquêtes démographiques et de santé ou des observatoires de population, montrent que les enfants dans les quartiers informels en Afrique subsaharienne sont en moins bonne santé (Günther \& Harttgen, 2012). Les études qui s'appuient sur ces enquêtes démographiques et de santé (EDS) intègrent un nombre limité des variables de l'environnement (accès à l'eau, gestion des eaux usées et des ordures). À Nairobi, où environ $60 \%$ de la population vit dans les quartiers informels, la mortalité est deux fois plus élevée que dans d'autres quartiers de la capitale (Egondi et al., 2013). Bocquier et ses collaborateurs (2011) mettent également en exergue une mortalité plus élevée chez les enfants des mères migrantes dans les quartiers informels à Nairobi. À Ouagadougou, par ailleurs, la mortalité des enfants est deux fois plus grande dans les quartiers non lotis à cause de conditions de vie insalubres (Rossier et al., 2011).

Du fait des inégalités dans l'accès à des services des soins préventifs, apparaît une variabilité en matière de santé entre les quartiers informels à l'intérieur d'une même ville et entre les villes des pays différents. En comparant la mortalité des enfants dans les quartiers non lotis de Ouagadougou et les slums de Nairobi, Soura et ses collaborateurs montrent que les slums de Nairobi sont moins vaccinés à cause des inégalités dans l'accès aux services de vaccination (Soura et al., 2014). Les conditions sanitaires particulièrement moins favorables aux quartiers informels ont été attribuées à l'insalubrité (Sy et al., 2011). Les facteurs environnementaux dans 
les quartiers exposent les populations urbaines à de multiples risques sanitaires. La recherche est peu avancée sur ce sujet, et varie fortement d'un contexte à l'autre. Chaque contexte urbain nécessite ainsi être décrit plus finement en recourant aux données pertinentes.

Dans cet article, nous décrirons finement le contexte sanitaire des quartiers formels (zones loties) et informels (zones non loties) à partir des données de l'Observatoire de Population de Ouagadougou (OPO). Nous testerons également si cette dichotomie loti/non loti constitue-t-elle une grille d'analyse pertinente à Ouagadougou. Nous analyserons ainsi les variations inter-quartiers (quartiers lotis comparés entre eux, quartiers non lotis comparés entre eux) et intra-quartiers.

\section{Méthodes}

\section{Contexte d'étude et échantillon}

Ouagadougou est la capitale du Burkina Faso, pays où environ $80 \%$ de la population active dépend de l'agriculture et de l'élevage (Yapi-Gnaoré et al., 2014). En 2007, la proportion estimée de personnes en ville ayant un niveau de consommation inférieur au seuil de pauvreté est d'environ 17\% (Burkina Faso et ONU, 2012). Peuplée d'environ deux millions d'habitants en 2010, Ouagadougou est l'une des plus importantes villes de l'Afrique de l'Ouest (INSD et ICF International, 2012). Entre 1960 et 2006, la population de la capitale a été multipliée par 15 (Guengant et al., 2009), le périmètre urbain est passé de $60 \mathrm{~km}^{2}$ en 1960 à $544 \mathrm{~km}^{2}$ en 2010 (Sanga, 2011), soit une multiplication par 9 en 50 ans. Ouagadougou constitue un exemple d'une ville africaine où la population a connu une croissance très rapide au cours des dernières années : son taux moyen annuel de croissance a été estimé à 7,6\% pour la période de 1985 à 2006 (Boyer, 2009); ce taux pourrait se situer autour de 9\% au cours de la prochaine décennie 2010-2020 (UN-Habitat, 2014). Ouagadougou dépasse de loin le groupe des villes à croissance très rapide du continent africain (Dar-es-Salaam, Kampala et Niamey) (ONU-Habitat, 2010). Le maintien d'un niveau élevé de croissance urbaine distingue nettement Ouagadougou de certaines autres villes de l'Afrique de l'Ouest comme Dakar et Abidjan qui, du fait de la baisse des migrations, ont connu un rythme de croissance plus modéré (Boyer, 2010). La croissance urbaine et l'évolution de l'espace urbain de la ville ralentissent les efforts de fournir les services urbains de base (adduction d'eau et assainissement) à l'ensemble de la population (Soura, 2009; Dos Santos, 2013).

La ville de Ouagadougou est marquée par un contraste entre le centre et la périphérie, et une dichotomie entre les quartiers lotis, où les terrains sont légalement acquis auprès de l'administration, et les quartiers non lotis ou informels (Boyer, 2010). Les quartiers lotis se caractérisent également par un réseau de rues régulières et la présence des services urbains de base 
(électricité, adduction d'eau, structures de santé, écoles, gestion des ordures, etc.) (Baragatti et al., 2009). Représentant 26\% de la surface potentiellement habitable de la ville de Ouagadougou (Boyer \& Delaunay, 2009), les quartiers non lotis, où par définition rien n'est entrepris par l'État en termes d'aménagement et d'urbanisme (Mondain et al., 2012), désignent les quartiers étant entrés dans le processus de lotissement. L'expansion des quartiers non lotis aux marges de la ville se justifie par la faiblesse de programme des logements sociaux, et par l'occupation spontanée ou l'achat de terrains auprès des chefs coutumiers (Boyer \& Delaunay, 2009). Cette dynamique d'extension résulte également du fait des migrants pauvres (généralement originaires des campagnes), des citadins majoritairement célibataires et des jeunes familles qui s'établissent dans ces zones non loties à densité variable pour accéder à la propriété foncière ou se servent de la construction comme une épargne (Boyer, 2010; Rossier et al., 2013). Environ un tiers de la population de la capitale vit en zones non loties caractérisées par la pauvreté et le manque de services urbains de base (Boyer \& Delaunay, 2009). Par ailleurs, le type de lotissement est corrélé aux caractéristiques du logement et aux biens possédés par les individus (Rossier et al., 2011). Dans les zones non loties par exemple, les maisons sont en banco en prévision d'une destruction les années à venir, alors que la plupart de maisons sont en ciment dans les zones loties (Rossier et al., 2011).

Les données de l'analyse proviennent de l'Observatoire de Population de Ouagadougou (OPO : un système de surveillance démographique). Depuis 2008, 1'OPO suit environ 85000 individus dans cinq quartiers localisés dans la périphérie nord de Ouagadougou (Rossier et al., 2012) : les quartiers lotis de Kilwin, de Tanghin, et les quartiers non lotis de Nioko 2, de Nonghin, et de Polesgo. Les quartiers de l'OPO ont été choisis pour cibler les populations les plus vulnérables pour lesquelles les données et les interventions dans le domaine de la santé sont prioritaires (Rossier et al., 2012).

L'analyse utilise exclusivement les données transversales de l'enquête sur la santé et celles de l'enquête sur les ménages, réalisées respectivement en 2010 et en 2009. L'enquête de santé a concerné 950 enfants de moins de 5 ans dans les cinq quartiers de l'OPO. L'enquête sur les ménages a, quant à elle, touché 81717 individus répartis dans 15925 ménages. L'analyse utilise également les données géo-localisées des 697 unités collectives d'habitation (UCH) ayant fait l'objet de l'enquête de santé et de deux dangers environnementaux: la proximité des tas d'ordures et des points d'eau stagnante. On entend par "danger environnemental" le potentiel que possède un agent d'origine biologique, chimique ou physique d'exercer un effet négatif sur la santé (Chevalier et al., 2003; Kientga, 2008; Dora et al., 2014). Les eaux stagnantes et les tas d'ordures sont des dangers environnementaux; ils peuvent ainsi représenter de risques de maladie pour les populations (Koné et 
al., 2014). L'analyse spatiale met en exergue ces deux variables. Les données sur les eaux stagnantes, géo-localisées de février à août 2010, sont d'autant plus pertinentes, puisque la présence des eaux stagnantes est en fonction de la saison en milieu sahélien (Mbaye et al., 2009). D'autres variables, telles que la proximité des latrines, constitueraient de grands dangers environnementaux (Graham \& Polizzotto, 2013), mais n'ont pas été considérées, parce qu'elles n'ont pas été géo-localisées. Néanmoins, la tendance est que les latrines sont plus proches des logements en zones non loties.

Le questionnaire enfant a permis de collecter des déclarations des mères (ou des gardiens) sur cinq symptômes déclarés de maladies des enfants apparus au cours des deux semaines précédant l'enquête (fièvre, diarrhée, toux, infections de la peau et des yeux), ainsi que des données anthropométriques. Le questionnaire au sujet du ménage a, par ailleurs, recueilli des données sur les facteurs de l'environnement immédiat tels que la présence d'un jardin potager ou celle des plantes extérieures dans l'UCH, etc.

\section{Méthodes d'analyse}

La première approche utilisée s'appuie sur les méthodes d'analyse spatiale basée sur la distance euclidienne dans le but d'évaluer la proximité des UCH aux espaces considérés « à risque » d'un point de vue sanitaire. En premier lieu, des cartes de distribution des $\mathrm{UCH}$ pour chaque quartier en rapport avec les zones tampons de 200 mètres de rayon autour de deux dangers environnementaux susceptibles d'exposer les enfants aux maladies - les tas d'ordures et les points d'eau stagnante - ont été réalisées au moyen du logiciel ArcGIS (version 10.2). Ces deux variables ont été retenues sur la base de la littérature et de leur disponibilité dans les données de l'OPO. Lorsque les ordures ne sont pas bien éliminées, elles peuvent être une source de prolifération de microbes, parasites et autres vecteurs de maladies (Kafando et al., 2013; Kouamé et al., 2014). Également, les eaux stagnantes peuvent favoriser la prolifération des moustiques vecteurs du paludisme surtout en saison pluvieuse (Piet Verdonschot et Besse-Lototskaya, 2014). Ces dangers environnementaux (tas d'ordures et points d'eau stagnante) peuvent ainsi favoriser la transmission des maladies, telles que le paludisme, les maladies diarrhéiques, les infections des yeux et de la peau (Unger, 2013; Walker et al., 2013). Les insectes tels que les moustiques, qui sont des vecteurs reconnus, sont limités dans leurs déplacements (Kientga, 2008), étant donné que les Anophèles parcourent des distances moyennes maximales allant de 200 à 35000 mètres (Piet Verdonschot \& Besse-Lototskaya, 2014). Le rayon de 200 mètres d'action a été retenu pour la réalisation des zones tampons en raison de la petite superficie des quartiers (pour plus de détail sur les méthodes, voir Chung et al. (2004) et Scott et Janikas (2010)). Nous faisons l'hypothèse que les moustiques parcourent cette distance en quête de proies et de lieux pour le 
dépôt des larves, et que les tas d'ordures et les points d'eau stagnante constituent des abris et limitent ainsi leur déplacement au rayon de 200 mètres. La littérature indique qu'il existe des prédilections variées pour des biotopes (marécages, trous d'arbres, ornières de roues, canaris, citernes et tanks, etc.) (Rogier, 2003; Guillaumot, 2005; Drabo et al., 2014). Ainsi, le rayon d'action concerne les Anophèles (paludisme) et non pas les Aedes aegypti (vecteurs de la dengue et de la fièvre jaune), car les Aedes aegypti ne se reproduisent pas dans des eaux stagnantes mais dans des eaux claires et propres (Ponlawat et al., 2005; Naing et al., 2011). Les Aedes aegypti sont également très casaniers et ne s'éloignent guère de plus de 100 mètres de leur gîte d'origine (Guillaumot, 2005). En second lieu, des cartes de proximité des UCH aux dangers environnementaux ont été produites pour saisir le risque d'exposition aux dangers liés à l'environnement urbain. Par la suite, des tests de Khi-deux ont été effectués pour apprécier la significativité des relations entre ces variables. De même, certains indicateurs mesurés au niveau du quartier (malnutrition de l'enfant, éducation de la mère) ont été calculés pour une comparaison plus fine avec les données sur la proximité des UCH aux dangers environnementaux. Enfin, des cartes de densité des noyaux (pour plus de détail sur la méthode de Kernel, voir Congdon (2013) pour des précisions sur cette méthode) ont été réalisées pour décrire la concentration des cinq symptômes de maladies déclarés à l'intérieur de chaque quartier. Les résultats de la concentration des symptômes n'auraient pas pu être biaisés, puisque les ménages ayant fait l'objet de l'enquête ont été tirés de manière systématique pour assurer la représentativité des zones géographiques de l'OPO.

Ensuite, une approche descriptive multivariée a caractérisé les quartiers par l'emploi de l'analyse factorielle des correspondances multiples (AFCM) à l'aide du logiciel SPAD (version 5.5). Il a été question d'étudier simultanément les relations entre tous les symptômes déclarés, ainsi que la malnutrition simple et sévère et les variables de l'environnement, afin de dégager les profils combinés à l'échelle du quartier. Une classification a ensuite été utilisée pour déterminer les groupes d'enfants à risque. Les variables liées à la morbidité des enfants et à l'environnement ont été considérées comme actives dans les analyses factorielles et de classification, c'est-à-dire, permettant de calculer les axes factoriels. Le quartier, le type de lotissement, le pourcentage des mères éduquées et la densité de la population ont été intégrés comme des variables illustratives, c'est-à-dire, ne participant pas au calcul des axes factoriels, pour lesquels on calcule des coordonnées factorielles qui auraient été affectées à une forme ayant la même répartition mais participant à l'analyse avec un poids négligeable. Au total, 15 variables actives d'intérêt avec 35 modalités associées et 4 variables illustratives de 13 modalités associées ont été intégrées dans l'analyse. 


\section{Variables}

La fièvre, la diarrhée, la toux, les infections des yeux et de la peau, ainsi que la malnutrition simple et sévère ont été retenues pour appréhender la morbidité chez les enfants. Des indices anthropométriques ont été calculés pour la malnutrition simple et sévère conformément aux normes de croissance de l'enfant définies par l'Organisation Mondiale de la Santé (OMS) de 2006. La malnutrition simple a été définie comme un rapport poids/âge entre -3 et $2 \mathrm{z}$-scores en dessous de la médiane indiquée par les normes de l'OMS (OMS, 2009; Buttarelli et al., 2013). Pour le rapport poids pour la taille, l'OMS recommande l'utilisation du seuil de -3 écarts types des normes OMS pour identifier les enfants ayant une malnutrition aiguë sévère (OMS, 2009).

Plusieurs variables clés susceptibles d'influencer la morbidité chez les enfants en milieu urbain africain ont été retenues, en se basant sur les études ayant souligné leur pertinence (Ngnikam et al., 2014; Sy et al., 2014) : l'approvisionnement en eau de boisson, la présence d'ordures dans le ménage, la gestion des ordures ménagères, la gestion des eaux usées, la nature du plancher intérieur, l'utilisation de la moustiquaire sur le lit dans lequel l'enfant dort, la présence d'un jardin potager et celle de plantes extérieures dans l'UCH. L'approvisionnement en eau de boisson a trois modalités : le robinet, les bornes fontaines et les autres sources (vendeurs d'eau, rivière, lac, canal d'irrigation et l'eau de pluie). La présence d'ordures dans le ménage, la présence d'un jardin potager, et celle des plantes extérieures dans l'UCH sont des variables dichotomiques, et elles ont été observées par les enquêteurs euxmêmes. La gestion des ordures ménagères regroupe trois modalités : le mode approprié (poubelle privée avec service de ramassage, bac public avec service de ramassage public ou centre de pré-collecte), les terrains vagues ou parcelles vides, et autres. La gestion des eaux usées regroupe deux modalités : les fosses septiques et la rue et autres (puits perdu, arrosage, etc.). Les modalités de la nature du plancher intérieur sont: le carrelage, le ciment, la terre et autres (bouse, bois ou autres végétaux). L'utilisation d'une moustiquaire sur le lit de l'enfant est captée pour la nuit avant l'enquête, et elle est une variable dichotomique.

Concernant le contexte de résidence, nous avons retenu le quartier (Kilwin, Tanghin, Nioko 2, Nonghin et Polesgo) et le type de lotissement (loti ou non loti). Des variables agrégées au niveau de quartier ont également été introduites : le pourcentage de mères éduquées (ayant été à l'école primaire ou plus) et la densité de population dans chaque quartier. Pour la densité démographique, les effectifs de la population sont rapportés à la superficie de chaque bloc d'habitation. La variable continue de densité obtenue a été discrétisée en trois classes standardisées selon la moyenne et l'écart-type après l'utilisation de la courbe gaussienne, ce qui a favorisé la représentation des valeurs moyennes : densité faible de population (moins de 4000 habitants par 
$\mathrm{km}^{2}$ ), densité moyenne de population (entre 4000 et 16000 habitants par $\mathrm{km}^{2}$ ) et densité élevée de population (plus de 16000 habitants par $\mathrm{km}^{2}$ ).

\section{Résultats}

\section{Analyse spatiale des données géo-localisées}

1. Distribution spatiale des unités collectives d'habitation autour des tas d'ordures et des points d'eau stagnante

Les Figures 1 et 2 montrent que les dangers environnementaux ne se répartissent pas de manière homogène entre les quartiers de l'OPO, de même que dans les UCH. En effet, on observe des différences importantes dans la distribution spatiale des tas d'ordures entre les quartiers. Tanghin (quartier loti) et Nioko 2 (quartier non loti) sont des quartiers où le nombre de tas d'ordures recensés est le plus élevé pendant l'enquête. Les points d'eau stagnante, quant à eux, sont plus répandus dans les quartiers lotis que dans les quartiers non lotis.

Si l'on considère une zone tampon de 200 mètres de rayon autour des tas d'ordures ou des points d'eau stagnante comme étant une zone potentiellement plus exposée, Nonghin, un des quartiers non lotis, apparaît plus exposé aux dangers environnementaux. Nonghin totalise un nombre élevé d'UCH dans les zones tampons, soit en moyenne environ 17,5 UCH dans les zones tampons autour de chaque tas d'ordures, et près de 6,5 $\mathrm{UCH}$ dans les zones tampons autour des points d'eau stagnante. Les analyses indiquent qu'il $\mathrm{y}$ a très peu de différences en termes d'exposition aux tas d'ordures et aux points d'eau stagnantes entre Kilwin et Polesgo, alors que Nioko 2 se caractérise par une faible exposition aux tas d'ordures (où en moyenne existent $6 \mathrm{UCH}$ dans les zones tampons) et aux points d'eau stagnante (2 $\mathrm{UCH}$ en moyenne se retrouvent dans les zones tampons) d'entre tous les quartiers de l'OPO, quel que soit le danger environnemental. Parmi les quartiers lotis, Kilwin se distingue nettement de Tanghin, avec une faible présence des UCH dans les zones tampons autour des tas d'ordures ou d'un point d'eau stagnante.

\section{Distances des unités collectives d'habitation aux tas d'ordures et points d'eau stagnante les plus proches}

La Figure 3 illustre la distance des UCH au tas d'ordures le plus proche dans les quartiers de l'OPO. Deux quartiers non lotis (Nioko 2 et Nonghin) se montrent avantagés en termes d'exposition au risque et les trois autres quartiers sont plus désavantagés (deux quartiers lotis et Polesgo).

En moyenne, les UCH des quartiers lotis (Kilwin et Tanghin, respectivement 102 mètres et 120 mètres) sont plus rapprochées des tas d'ordures comparativement aux UCH des quartiers non lotis. Parmi les quartiers non lotis, Nioko 2 se démarque par des $\mathrm{UCH}$, en moyenne, plus 
éloignées des tas d'ordures (156 mètres). Les différences entre les quartiers à ces égards sont significatives au seuil de $1 \%$.

La distance des UCH au point d'eau stagnante le plus proche offre quasiment une configuration analogue à la Figure 3 (cf. Figure 4). Les quartiers non lotis se révèlent avantagés en termes de risque et les deux quartiers lotis et Polesgo sont plus défavorisés. Les UCH des quartiers lotis sont plus rapprochées des points d'eau (Kilwin et Tanghin, respectivement 152 mètres et 258 mètres). Parmi les quartiers non lotis, Nioko 2 se distingue par des UCH, en moyenne, plus éloignées des points d'eau (309 mètres). Ces différences entre les quartiers sont significatives au seuil de $1 \%$.

Pour mieux apprécier les résultats concernant la proximité aux dangers environnementaux, le Tableau 1 met en parallèle ces données avec certaines caractéristiques au niveau du quartier. L'examen du tableau montre que les quartiers lotis bien que vulnérables en termes d'exposition aux deux dangers environnementaux présentent des indicateurs de malnutrition de l'enfant et d'éducation de la mère plus favorables par rapport à Nioko 2 et Nonghin. Le pourcentage des enfants avec malnutrition modérée et celui des enfants avec malnutrition sévère sont particulièrement plus faibles à Kilwin (quartier loti) comparés à ceux observés dans les quartiers non lotis. Également, le pourcentage des mères éduquées à Kilwin (37\%) et à Tanghin (23\%) est nettement plus important dans ces deux quartiers lotis que dans les quartiers non lotis suivis par l'OPO. 
Figure 1: Distribution des unités collectives d'habitation autour de tas d'ordures

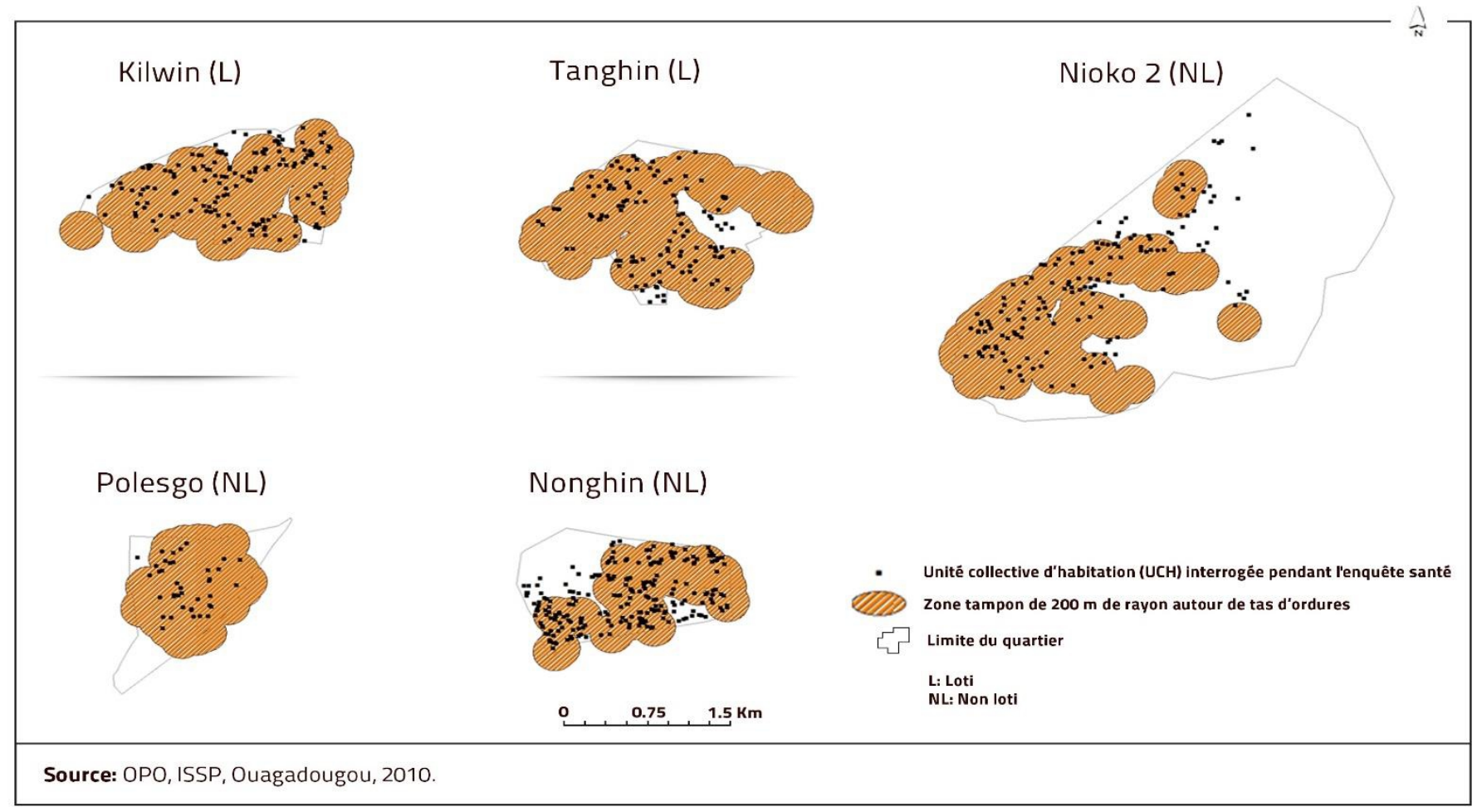


Figure 2: Distribution des unités collectives d'habitation autour de points d'eau stagnante

Kilwin (L)


Tableau 1. Proximité des dangers environnementaux en comparaison avec certaines caractéristiques des quartiers (OPO, 2010)

\begin{tabular}{|l|c|c|c|c|c|}
\hline Quartiers & $\begin{array}{l}\text { Distance des } \\
\text { UCH au tas } \\
\text { d'ordures le } \\
\text { plus proche (en } \\
\text { m) }\end{array}$ & $\begin{array}{l}\text { Distance des au point } \\
\text { UCH leau plus } \\
\text { d'soche (en m) } \\
\text { procen }\end{array}$ & $\begin{array}{l}\text { Enfants avec } \\
\text { malnutrition } \\
\text { modérée (en } \\
\text { \%) }\end{array}$ & $\begin{array}{l}\text { Enfants avec } \\
\text { malnutrition } \\
\text { sévère (en } \\
\text { \%) }\end{array}$ & $\begin{array}{l}\text { Mères } \\
\text { éduquées } \\
\text { (en \%) }\end{array}$ \\
\hline $\begin{array}{l}\text { Kilwin } \\
\text { (L) }\end{array}$ & 102 & 152 & 13,5 & 3,4 & 37,1 \\
\hline $\begin{array}{l}\text { Tanghin } \\
\text { (L) }\end{array}$ & 120 & 258 & 25,2 & 5,2 & 23,4 \\
\hline $\begin{array}{l}\text { Polesgo } \\
\text { (NL) }\end{array}$ & 64 & 167 & 18,1 & 4,5 & 4,9 \\
\hline $\begin{array}{l}\text { Nonghin } \\
\text { (NL) }\end{array}$ & 127 & 268 & 16,7 & 3,0 & 20,9 \\
\hline $\begin{array}{l}\text { Nioko } 2 \\
\text { (NL) }\end{array}$ & 156 & 309 & 21,7 & 7,8 & 13,7 \\
\hline
\end{tabular}

Note: L : loti ; NL : non loti ; UCH: Unité collective d'habitation.

\section{Analyse de concentration des symptômes déclarés dans les quartiers de l'OPO}

La Figure 5 présente les surfaces de densité indiquant les lieux particulièrement vulnérables où les symptômes déclarés de maladies sont concentrés dans les zones de l'OPO. L'analyse des densités des noyaux montre une répartition inégale de la concentration des symptômes à l'intérieur d'un même quartier, de même entre les différents quartiers. Les concentrations des symptômes sont non seulement observables dans les quartiers non lotis, mais également dans les quartiers lotis. Parmi les quartiers non lotis, plus particulièrement Nonghin et, dans une moindre mesure, Nioko 2 présentent une multitude de zones de concentration des symptômes, et se présentent donc comme particulièrement vulnérables. Ces zones de concentration de symptômes forment des îlots de manière éparse. Les quartiers lotis, quant à eux, présentent des foyers de concentration des symptômes relativement moins denses. De même, Polesgo, bien que zone non lotie, se révèle moins vulnérable en se distinguant par une concentration faible des symptômes. 
Figure 3: Distance des unités collectives d'habitation aux tas d'ordures les plus proches

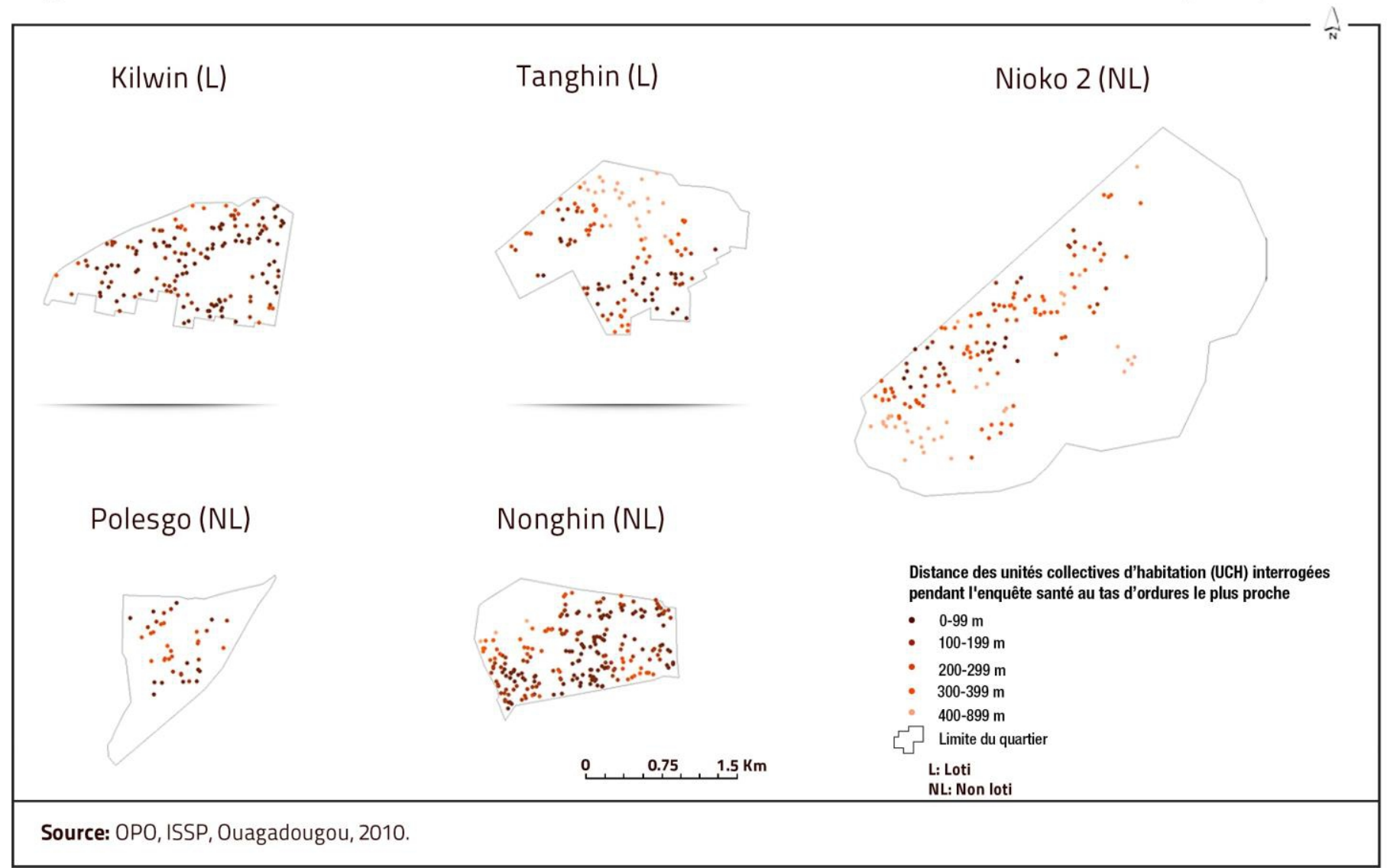


Figure 4: Distance des unités collectives d'habitation aux points d'eau stagnante les plus proches

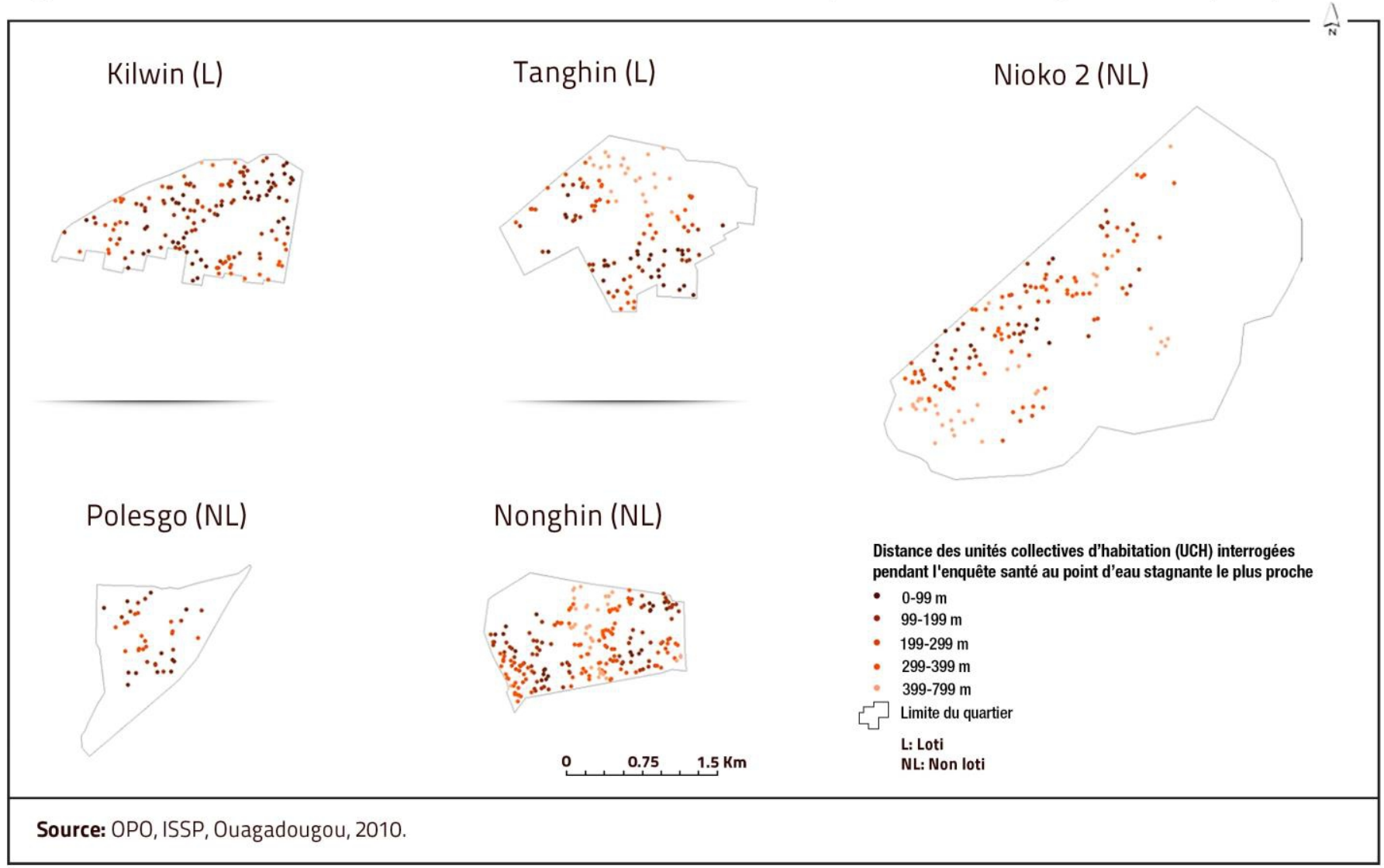


Figure 5: Répartition de densités des noyaux pour les symptômes dans les zones de l'OPO

Kilwin (L) Nioko 2 (NL)




\section{$\checkmark$ Analyses factorielles et de classification 1. Interprétation des axes factoriels}

L'objectif des analyses factorielles est d'étudier les quartiers et les groupes d'enfants à risque de maladies. Les trois premiers axes factoriels (1, 2 et 3) contribuent respectivement à $12,3 \%, 9,6 \%$ et $7,3 \%$, soit environ $29 \%$ de l'inertie totale. L'axe 1 ordonne les enfants selon l'accès à l'eau et la caractéristique du plancher intérieur (respectivement $28,2 \%$ et $17,5 \%$ ). L'axe 2 est constitué principalement par les symptômes (fièvre, toux et diarrhée, avec respectivement $27,5 \%, 24,1 \%$ et 9,5\%), tandis que l'axe 3 est constitué par la présence de jardin potager dans l'UCH (26,2\%), la gestion des eaux usées et des ordures ménagères (avec respectivement $25,6 \%$ et 23,8\%). Ainsi, 1'axe 1 peut être interprétée comme l'axe de la pauvreté du fait qu'elle est captée par les caractéristiques de l'habitat (accès à l'eau, nature du plancher, etc.) et les biens d'équipements possédés par le ménage (Sheppard et al., 2009), l'axe 2 comme celui de morbidité, et l'axe 3 comme celui de l'assainissement.

\section{Profil des quartiers par rapport aux maladies et à l'environnement}

Le Graphique 1 illustre le premier plan factoriel (axes 1 et 2) dans lequel deux grands groupes de quartiers s'opposent diamétralement. Dans le premier groupe à gauche se retrouvent les quartiers non lotis (Nioko 2, Nonghin et Polesgo), qui sont soumis à une densité de population variable (faible et élevée) et un faible pourcentage de mères éduquées (moins d'une mère sur deux). En général, leurs enfants présentent plus de signes de maladies et utilisent moins de moustiquaires. Les ménages s'approvisionnent en eau aux bornes-fontaines ou aux autres sources (vendeurs d'eau, rivière, lac, canal d'irrigation et eau de pluie) et les ordures sont présentes dans les ménages et jetées dans des terrains vagues ou des parcelles vides. Le plancher des logements dans ces quartiers est majoritairement fait en terre et autres (bouse, bois ou autres végétaux) et des jardins potagers sont entretenus dans les UCH.

Dans le deuxième groupe, figurent les quartiers lotis (Kilwin et Tanghin). Dans l'ensemble, ces quartiers sont caractérisés par une densité de population moyenne et un pourcentage relativement élevé de mères éduquées (entre 33 et $50 \%$ de mères). Les enfants y ont moins de symptômes déclarés et utilisent plus des moustiquaires. Les ménages possèdent plus souvent un robinet dans la cour ou le logement. Les ordures ne sont pas autant présentes dans les ménages, et un mode approprié de gestion des ordures (poubelle privée avec service de ramassage, bac public avec service de ramassage public ou centre de pré-collecte) est plus souvent utilisé. Ces logements ont tendance à posséder des fosses septiques, à avoir des planchers en carrelage, et des plantes extérieures sont entretenues dans les UCH. 
Il ressort de l'analyse que l'ensemble des pathologies se concentre dans les quartiers non lotis et, plus particulièrement, à Nioko 2. De plus, les résultats mettent clairement en évidence des relations entre les maladies et la malnutrition modérée et sévère: plus les enfants sont malnutris, plus nombreux sont les symptômes déclarés. Il se dégage également de l'analyse que le type d'approvisionnement en eau de boisson, l'assainissement, la nature du plancher et l'occurrence de maladies sont des variables importantes par rapport à la contribution de leurs modalités. Ces variables sont donc à considérer pour des analyses plus minutieuses.

Graphique 1. Premier plan factoriel d'une analyse des correspondances multiples sur 19 variables, 48 modalités et 950 enfants $(O P O, 2010)$

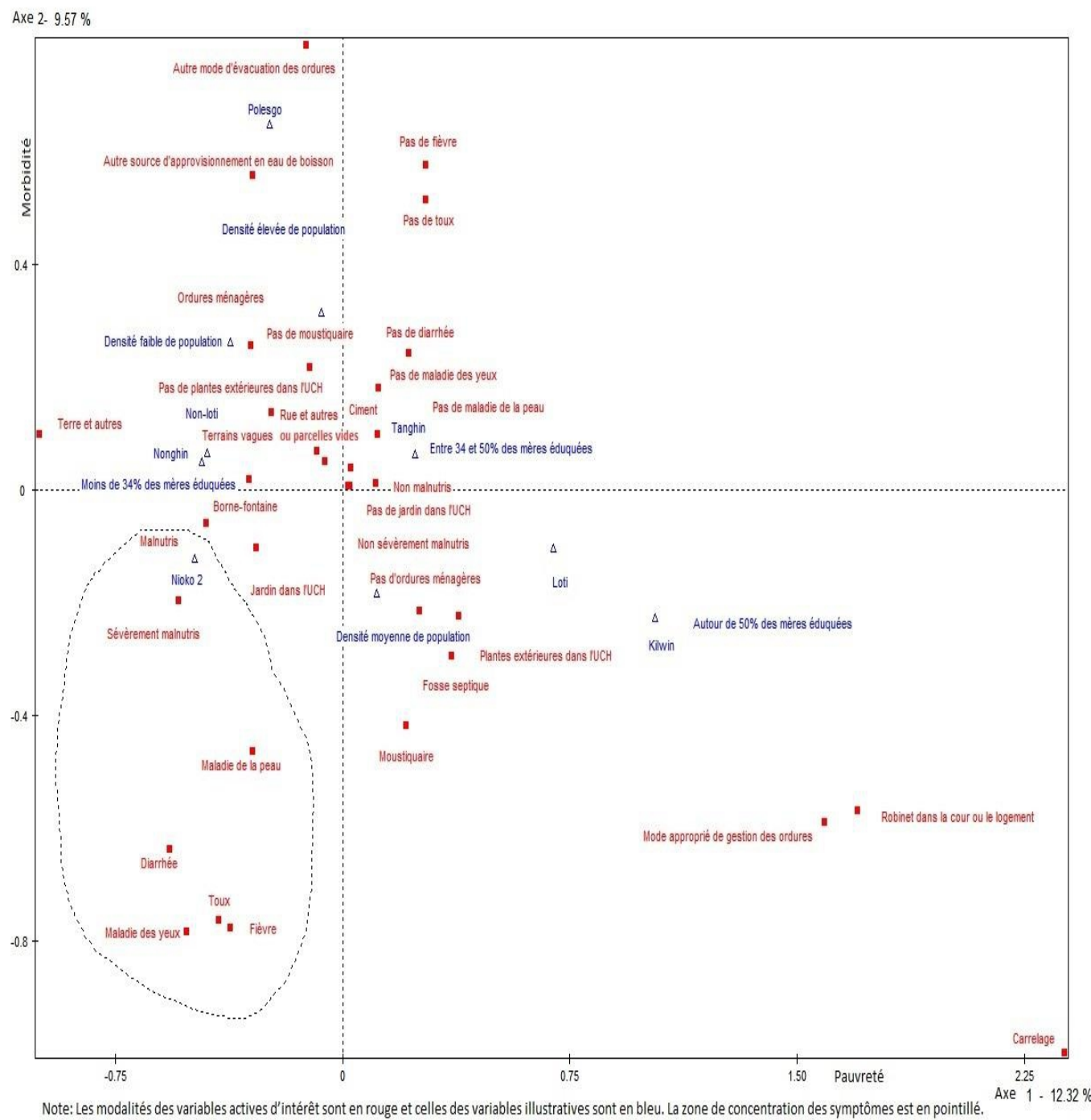




\section{$\checkmark$ 3. Profil des groupes d'enfants à risque de maladies environnementales}

Par souci d'avoir une partition (classe issue d'analyse de classification) interprétable et des groupes d'effectifs raisonnables, nous avons choisi d'étudier deux partitions, notamment à deux et trois groupes. L'analyse de la partition en deux et trois groupes a conduit à retenir la partition en trois groupes. Le Graphique 2 projette la partition en trois groupes sur un plan factoriel. Le premier groupe (groupe 1) totalise environ 61\% de l'échantillon. La fièvre, la diarrhée, la toux, les infections des yeux, la malnutrition modérée sont déclarées chez les enfants. Les enfants ne sont pas sévèrement malnutris et n'ont pas d'infections de la peau. Les enfants appartiennent aux ménages qui s'approvisionnent aux bornes-fontaines ou aux autres sources (vendeurs d'eau, rivière, lac, canal d'irrigation et eau de pluie). Le plancher des logements est en ciment. Les ordures sont présentes dans les ménages, elles sont parfois jetées dans les terrains vagues ou les parcelles vides. Les eaux usées sont déversées dans la rue et autres (puits perdu, arrosage, etc.). Les jardins potagers ne sont pas entretenus dans les UCH. Le groupe 1 est celui des enfants « moyennement à risque » de maladies.

Le deuxième groupe (groupe 2) concentre environ $25 \%$ des enfants de l'échantillon. La diarrhée, la toux, les infections de la peau, la malnutrition modérée et sévère sont plus marquées chez les enfants de ce groupe, tandis que la fièvre et les infections des yeux y sont plus rares. Les enfants ne dorment pas sous une moustiquaire. Les ménages s'approvisionnent aux bornes-fontaines, de même qu'aux autres sources (vendeurs d'eau, rivière, lac, canal d'irrigation et eau de pluie). Les ordures sont présentes dans les ménages. Les ménages possèdent des fosses septiques et utilisent la rue et autres modes d'évacuation des ordures. Le plancher des logements est en terre et autres (bouses, bois ou autres végétaux). Les jardins potagers sont entretenus dans les UCH, alors que les plantes extérieures ne le sont pas. Le groupe 2 est celui des enfants « plus à risque » de maladies.

Le troisième groupe (groupe 3) totalise environ $15 \%$ de l'échantillon. Les enfants de ce groupe ne présentent pas de signes de maladies (fièvre, diarrhée, toux, infections des yeux et de la peau, malnutrition modérée et sévère). Les enfants dorment sous une moustiquaire. Les ménages disposent de planchers en carrelage, s'approvisionnent au robinet dans la cour ou le logement, et ils ont des fosses septiques. Les ordures ne sont pas présentes dans les ménages, puisqu'ils utilisent le mode approprié de gestion des ordures. Les plantes extérieures sont entretenues dans les $\mathrm{UCH}$, tandis que les jardins potagers ne le sont pas. Le groupe 3 est celui des enfants « faiblement à risque $\gg$ de maladies.

Les enfants se répartissent de manière inégale selon le quartier à partir de l'analyse de la classification (Tableau 2). Les enfants issus 
de Nonghin et Nioko 2, deux quartiers non lotis sont plus représentés dans les groupes 1 et 2 (avec respectivement plus de $40 \%$ et plus de $20 \%$ dans chaque groupe) comparativement aux enfants de Polesgo faiblement représentés dans ces groupes. À l'opposé de ces enfants, ceux issus de Kilwin et Tanghin sont majoritaires dans le groupe 3 (respectivement $76 \%$ et $22 \%$ ).

Tableau 2. Répartition des enfants (en pourcentage) en trois groupes issus de l'analyse de classification, OPO, 2010

\begin{tabular}{|l|c|c|c|c|}
\hline Quartiers & $\begin{array}{l}\text { Enfants «plus à } \\
\text { risque » de maladies } \\
(\%)\end{array}$ & $\begin{array}{l}\text { Enfants } \\
\text { « moyennement à } \\
\text { risque de maladies » } \\
(\%)\end{array}$ & $\begin{array}{l}\text { Enfants « faiblement } \\
\text { à risque de maladies » } \\
(\%)\end{array}$ & Total (\%) \\
\hline Kilwin (L) & 14,5 & 13,0 & 75,5 & 22,5 \\
\hline Tanghin (L) & 14,1 & 16,1 & 22,3 & 16,5 \\
\hline Polesgo (NL) & 6,4 & 9,0 & 0,0 & 7,1 \\
\hline $\begin{array}{l}\text { Nonghin } \\
\text { (NL) }\end{array}$ & 41,0 & 41,2 & 0,0 & 35,2 \\
\hline Nioko 2 (NL) & 23,9 & 20,6 & 2,2 & 18,7 \\
\hline Total & 100,0 & 100,0 & 100,0 \\
\hline
\end{tabular}

La partition en 3 groupes révèle, au-delà de la dualité «loti-non loti», une ségrégation entre les enfants en termes de santé environnementale. On voit que même si le groupe "plus à risque » de maladies (groupe 3) renferme les enfants de différents quartiers avec une primauté du milieu non loti, environ un quart des enfants de Kilwin et près de $80 \%$ des enfants de Tanghin se retrouvent dans les groupes 1 et 2 , groupes les plus à risque de maladies. Ces résultats indiquent clairement que les risques environnementaux ne concernent pas essentiellement les quartiers non lotis, mais touchent plutôt l'ensemble des quartiers en outrepassant la dichotomie loti/non loti. 
Graphique 2 : Projection des groupes sur le plan factoriel d'une analyse des correspondances multiples, OPO 2010

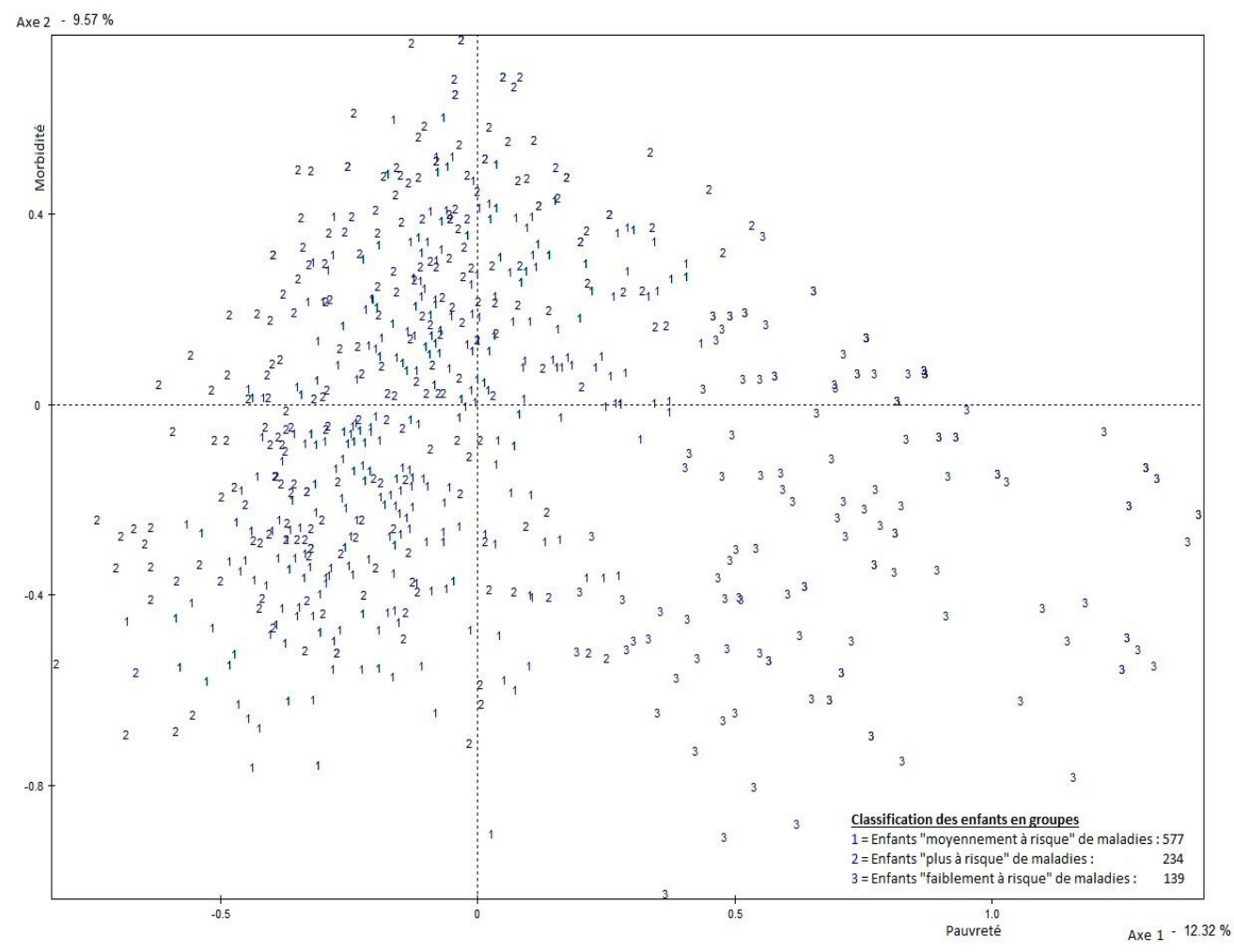

\section{$\checkmark$ Discussion et conclusion}

Ce travail avait pour objectif de décrire les quartiers les plus à risque de morbidité (fièvre, diarrhée, toux, infections de la peau et des yeux) dans les zones de l'OPO. L'une des forces majeures de l'étude réside, à travers un regard croisé, dans l'utilisation des analyses spatiales, factorielles et de classification pour une description plus fine du contexte sanitaire des quartiers formels et informels en milieu urbain africain. Notamment l'analyse de classification, par rapport à l'analyse factorielle (qui confirme la pertinence de la dichotomie classique loti/non loti), a permis de dépasser la dichotomie loti/non loti, en mettant l'accent sur une hétérogénéité en matière de santé environnementale. De plus, les données recueillies par l'OPO sur l'environnement immédiat et les symptômes sont plus diversifiées que celles des enquêtes démographiques et de santé, et le fait que trois des co-auteurs aient passé beaucoup de temps à travailler dans les zones de l'OPO offre la possibilité de mieux discuter ces données et la façon d'interpréter les résultats (observation sur le terrain des tas d'ordures, des points d'eau stagnante, etc.). 
En revanche, l'une des faiblesses des données pourrait concerner les problèmes liés à la définition statistique d'un quartier de résidence qui ne rime pas forcément avec l'espace où un individu vit (comme c'est le cas des quartiers non lotis), et le manque de données susceptibles de caractériser les autres espaces de socialisation (Andersson \& Musterd, 2010). Une autre limite pourrait concerner le biais de sélection des quartiers du fait que l'étude n'est pas exhaustive au niveau des quartiers de Ouagadougou. Les quartiers ont été choisis pour cibler les populations les plus vulnérables, et devaient être proches les uns des autres afin de réduire les coûts de collecte des données et des interventions à tester (Rossier et al., 2012). On peut également penser que d'autres limites de l'article pourraient concerner le choix d'un rayon de 200 mètres pour les analyses spatiales, l'utilisation de la distance euclidienne (sans possibilité d'analyser les barrières), et le biais de déclaration relatif aux symptômes de maladies (non médicalement vérifiés) (Al-Eissa et al., 2000). Toutefois, le rayon d'action de 200 mètres ne saurait affecter la fiabilité des résultats, puisque l'environnement urbain (avec la densité de la population et des animaux) est susceptible de réduire le déplacement des vecteurs de maladies notamment les moustiques (Kientga, 2008). Les moustiques sont plus reconnus étant "volants" au stade mature; de ce fait la distance euclidienne intègre bien cette dimension (Guillaumot, 2005), bien qu'elle n'autorise pas l'analyse des barrières physiques. Également, le fait que les mères éduquées ne confrontent pas une occurrence plus élevée de symptômes que celles non éduquées renforce la confiance accordée aux résultats.

Les résultats de l'analyse spatiale donnent à penser que les quartiers lotis sont plus à risque de maladies que les quartiers non lotis en termes de proximité des dangers environnementaux (tas d'ordures et point d'eau stagnante les plus proches) et donc de la possibilité de contamination. Pourtant, les quartiers lotis sont relativement moins touchés par des maladies. L'analyse spatiale apporte un nouvel éclairage sur l'exposition potentielle aux dangers environnementaux en milieu urbain. Vivre près d'une eau stagnante ou d'un tas d'ordures suppose a priori un risque de maladies plus élevé comme conséquence de l'exposition aux dangers environnementaux (Kouamé et al., 2014). Le fait d'avoir plus de mères éduquées, une proportion relativement faible d'enfants malnutris, l' accès à l'eau potable et à l'assainissement, ainsi que la nature du sol dans les quartiers lotis expliqueraient une occurrence faible des maladies en zones loties (El-Fadel et al., 2014). La proximité des dangers environnementaux dans les zones loties, notamment les tas d'ordures peut s'expliquer par l'inefficacité des services de ramassage ou la nonadhésion aux services de ramassage dans les quartiers lotis (Mpakam et al., 2006). Lorsque les ordures ne sont pas collectées, les gens trouvent toujours d'autres moyens de s'en débarrasser en les jetant dans des espaces rares (Aurah, 2013). En revanche, en milieu non loti, les concessions ont amplement 
d'espace entre elles (Bologo et al., 2010). Cela pourrait offrir la possibilité aux populations d'éloigner les ordures et réduire la propagation des maladies infectieuses.

En indiquant que les risques environnementaux ne sont pas uniformément répartis dans les zones de l'OPO, les analyses mettent d'abord en évidence une forte hétérogénéité inter-quartier (dichotomie loti/non loti). Les résultats de l'analyse pourraient s'expliquer par la densité du bâti des zones non loties habitées plus élevée par rapport à celle des zones loties habitées. La trame urbaine est plus resserrée (avec des parcelles plus petites) en zones non loties habitées (Boyer \& Delaunay, 2009), ce qui fait que les latrines sont plus proches des logements. Également, comparées aux zones loties, la densité des personnes par pièce habitable est plus importante en zones non loties : il y a 2 personnes par pièce habitable en zones non loties contre 1,7 personne par pièce habitable en zones loties (Rossier et al., 2011). Ces résultats confirment ceux des recherches antérieures qui considèrent le lotissement comme un marqueur de vulnérabilité (Vallée et al., 2006). À Nouakchott (Mauritanie) par exemple, une étude réalisée par Sy et al. (2011) suggère classiquement une homologie entre les risques sanitaires et les conditions environnementales défavorables des quartiers. L'accès à l'eau potable, la gestion des eaux usées et la collecte des ordures ménagères sont ainsi évoqués pour expliquer les risques sanitaires entre les quartiers (Sy et al., 2011). Néanmoins, la distinction socio-spatiale doit être dépassée parce que plus d'un quart des enfants de Kilwin et surtout $80 \%$ des enfants de Tanghin se retrouvent dans les groupes plus à risque de maladies.

Au-delà de la dichotomie loti/non loti, les analyses suggèrent une hétérogénéité intra-quartier en milieu loti. L'hétérogénéité en milieu loti pourrait s'expliquer par des différences démographiques, économiques et historiques entre Kilwin et Tanghin. Tanghin enregistre une croissance démographique plus importante (5,3\%) que Kilwin (2,3\%) (Rossier et al., 2012). Les ménages à Tanghin disposent de peu de ressources et leur quartier a été peuplé plus tôt que Kilwin (Rossier et al., 2012). Bien que loti, Tanghin est en effet un quartier dégradé et atypique où se pratique le maraichage urbain(Fournet et al., 2013) ; il est un quartier plus pauvre par rapport à Kilwin (Rossier et al., 2011) : il n'est pas représentatif de l'ensemble des quartiers lotis de Ouagadougou. Ce constat renforce bien l'idée d'un « entre-deux » qui évoque la complexité de quartiers que l'on a tendance à définir en opposition à l'espace légal (Clerc, 2011). Le caractère évolutif de la ville est une autre dimension qui rend aléatoire toute tentative de classification définitive des quartiers, car un quartier formel peut se dégrader, se taudifier et devenir insalubre (Gossé, 1991).

Les analyses révèlent également une démarcation intra-quartier en milieu non loti. Les quartiers non lotis (Nioko 2, Nonghin), bien qu'éloignés, 
se ressemblent et sont plus à risque de maladies. Nonghin et Nioko 2 forment le « prototype » du milieu non loti en se démarquant de Polesgo par des risques sanitaires plus élevés. Contrairement à Polesgo, Nonghin et Nioko 2 sont caractérisés par la présence de grands trous (favorisant l'accumulation d'eau) et de voies inondables, le manque d'un centre de santé et des associations pouvant sensibiliser les populations sur la prévention des maladies (Bologo et al., 2010). Ces résultats sont similaires à ceux des études portant sur plusieurs pays indiquant que les zones informelles, caractérisées par un accès insuffisant à des installations d'assainissement et d'eau de boisson propre, sont confrontées à une multitude de problèmes de santé liés à l'environnement (Fink et al., 2013; Kimani-Murage et al., 2014). Toutefois, même si les niveaux de morbidité sont nettement plus élevés dans les zones informelles que dans les zones formelles, ils sont plus faibles que dans les zones rurales dans la plupart des pays (Günther \& Harttgen, 2012). Bien qu'il soit un ancien village récemment phagocyté par la ville et entouré par des quartiers nouvellement lotis, Polesgo est relativement mieux doté en infrastructures étatiques (un dispensaire et des écoles primaires) par rapport aux deux autres zones non loties (Bologo et al., 2010). Polesgo dispose également d'un dynamisme des organisations communautaires qui sensibilisent les populations sur les maladies et qui interviennent dans la salubrité et l'hygiène (Bologo et al., 2010).

Bien souvent, la tendance est à généraliser les attributs des quartiers (les quartiers sont dotés d' infrastructures publiques et ils sont plus riches), et il n'est pas rare que les études trouvent un effet de quartier important, même après contrôle des autres facteurs (Bacqué \& Fol, 2007). Le présent article observe une hétérogénéité au sein des quartiers, des désavantages certains dans les quartiers lotis (proximité des contaminants, densité), mais tout de même dans l'ensemble des avantages en termes de morbidité. L'occupation des quartiers non lotis, comme le montrent certaines études, constitue une stratégie d'accès à la propriété foncière et au logement à long terme (Dos Santos, 2005). De jeunes ménages dynamiques avec certaines ressources qui visent une mobilité sociale ascendante peuvent opter de s'installer dans un quartier non loti, afin d'accéder à la propriété (Rossier et al., 2011; Soura et al., 2014). Bien qu'il y ait des différences loti/non loti qui demeurent pertinentes, on observe la présence des quartiers atypiques dans tous les milieux de résidence loti/non loti (cas de Polesgo avec certains avantages et celui de Tanghin, loti mais très dégradé : $80 \%$ des enfants de Tanghin se retrouvent dans les groupes plus à risque de maladies). La dichotomie loti/non loti n'est pas tout à fait absolue, et elle est donc nuancée compte tenu de l'hétérogénéité en matière de santé environnementale (notamment en zones non loties). Ce constat interpelle à mieux penser le concept de non loti dans les villes en Afrique de l'Ouest francophone, ou de slum dans d'autres villes 
africaines. Il interpelle également la pluralité des facteurs qui influencent la morbidité des enfants et la problématique de découpage plus minutieux des quartiers trop hétérogènes, ainsi que le choix du niveau d'agrégation $(\mathrm{UCH}$, ménage). Cela soulève de ce fait des questions liées aux données (types de données, difficultés à les collecter et à les utiliser dans les grands systèmes d'enquêtes plus standards), de même que le besoin de recourir plus en détail aux analyses multivariées sur les déterminants des problèmes de santé particuliers pour mieux comprendre les effets des facteurs de l'environnement spécifiques. Les analyses multivariées permettront ainsi de prendre en compte l'effet des facteurs d'influence que l'on contrôlera simultanément. Il serait ainsi intéressant d'analyser les effets des facteurs environnementaux immédiats contrôlés par les variables démographiques et socio-économiques sur les symptômes de maladies.

L'article suggère aussi le besoin d'un échantillon plus grand pour obtenir une estimation plus exacte d'une caractéristique qui varie beaucoup d'une unité à l'autre. Aussi, serait-il intéressant de collecter des données sur des facteurs de contagion environnementale plus large. Il faudrait donc recueillir des données sur la direction et vitesse du vent, afin de contrôler leurs effets sur des symptômes liés à l'environnement immédiat. La non-prise en compte de ces éléments pourrait entraîner une sous-estimation des erreurs standards de mesure. Le déplacement des populations ou des animaux entre les quartiers, etc. doivent également être considérés pour leur rôle dans la propagation des maladies.

\section{Remerciements}

La réalisation de ce travail a été possible grâce au financement du Programme Population et Santé en Afrique subsaharienne (PPSA-financé par la Fondation Bill et Melinda Gates) et à l'encadrement du Laboratoire Population-Environnement-Développement (LPED) de l'Institut de recherche pour le développement (IRD) et de l'Université de Montréal. Nous tenons aussi à exprimer nos remerciements à l'Institut supérieur des sciences de la population (ISSP) de l'Université de Ouagadougou pour les données mises à notre disposition. Finalement, nos remerciements vont au groupe des professeurs Clémentine Rossier, Solène Lardoux et Valery Ridde pour leurs commentaires.

\section{References:}

1. Al-Eissa, Y. A., Al-Sanie, A. M., Al-Alola, S. A. et al. (2000). «Parental perceptions of fever in children», Annals of Saudi Medicine, 20, 4: 202-205.

2. Andersson, R. \& Musterd, S. (2010). «What scale matters ? Exploring the relationships between individuals social poistion, neighbourhood 
context and the scale of neighbourhood», Geografiska Annaler: Series B, Human Geography, 92, 1: 23-43.

3. Aurah, C. M. (2013). «Assessment of Extent to Which Plastic Bag Waste Management Methods Used in Nairobi City Promote Sustainability», Nature, 1, 4: 96-101.

4. Bacqué, M.-H. \& Fol, S. (2007). «Effets de quartier: enjeux scientifiques et politiques de l'importation d'une controverse», dans J.Y. Authier, M.-H. Bacqué et F. Guérin-Pace (dir.), Le quartier. La découverte: 181-193.

5. Baragatti, M., Fournet, F., Henry, M. C. et al. (2009). «Social and environmental malaria risk factors in urban areas of Ouagadougou, Burkina Faso», Malaria Journal, 8, 1: 1-14.

6. Bocquier, P., Madise, N. J. \& Zulu, E. M. (2011). «Is there an urban advantage in child survival in sub-Saharan Africa? Evidence from 18 countries in the 1990s», Demography, 48, 2: 531-558.

7. Bologo, E., Moundain, N., Randall, S. et al. (2010). Morphologie sociale du quartier de Nioko II. Ouagadougou, $20 \mathrm{p}$.

8. Bologo, E., Moundain, N., Randall, S. et al. (2010). Morphologie sociale du quartier de Polesgo. Ouagadougou, $22 \mathrm{p}$.

9. Boyer, F. (2010). "Croissance urbaine, statut migratoire et choix résidentiels des Ouagalais. Vers une insertion urbaine ségrégée ?», Revue Tiers Monde, 1, 201: 47-64.

10. Boyer, F. \& Delaunay, D. (2009). Peuplement de Ouagadougou et développement urbain. Ouagadougou, IRD, $250 \mathrm{p}$.

11. Burkina Faso \& ONU (2012). Cadre d'accéleration des OMD (CAO) au Burkina Faso. Éliminer l'extreme pauvreté, 11 p.

12. Buttarelli, E., Chapuis-Lucciani, N., Badiane, N. S. et al. (2013). «État nutritionnel du jeune enfant (0-3 ans) à Pikine-Dagoudane en milieu urbain sénégalais», Bulletins et mémoires de la Société d'anthropologie de Paris, 25, 1-2: 83-98.

13. Chen, M., Zhang, H., Liu, W. et al. (2014). «The Global Pattern of Urbanization and Economic Growth: Evidence from the Last Three Decades», PloS One, DOI: 10.1371/journal.pone.0103799.

14. Chevalier, P., Cordier, S., Dab, W. et al. (2003). «Santé environnementale», dans M. Gérin, P. Gosselin, S. Cordieret al (dir.), Environnement et santé publique: fondements et pratiques. Paris, Edisem/Tec et Doc, Acton Vale: 59-86.

15. Chung, K., Yang, D.-H. \& Bell, R. (2004). «Health and GIS: toward spatial statistical analyses», Journal of Medical Systems, 28, 4: 349360 . 
16. Clerc, V. (2011). «Du formel à l'informel dans la fabrique de la ville, politiques foncières et marchés immobiliers à Phnom Penh», dans (dir.), Espace et sociétés. 63-79.

17. Congdon, P. (2013). «Spatially interpolated disease prevalence estimation using collateral indicators of morbidity and ecological risk», International Journal of Environmental Research and Public Health, 10, 10: 5011-5025.

18. Dora, C., Haines, A., Balbus, J. et al. (2014). «Indicators linking health and sustainability in the post-2015 development agenda», The Lancet, 381, 9866: 566-574.

19. Dos Santos, S. (2005). Koom la viim : enjeux socio-sanitaires de la quête de l'eau à Ouagadougou (Burkina Faso). Département de démographie. Montréal, Université de Montréal, Thèse de doctorat, $182 \mathrm{p}$.

20. Dos Santos, S. (2013). «L'accès à l'eau courante des femmes migrantes à Ouagadougou: durabilité de l'accès et effet sur la survie des enfants», VertigO-la revue électronique en sciences de l'environnement, DOI: 10.4000/vertigo.13289.

21. Drabo, K. M., Sawadogo, A., Laokri, S. et al. (2014). «Pratiques de prévention antipaludique dans les zones périurbaines de deux districts sanitaires du Burkina Faso», Santé Publique, 26, 5: 679-684.

22. Egondi, T., Kyobutungi, C., NG, N. et al. (2013). "Community perceptions of air pollution and related health risks in Nairobi Slums», International Journal of Environmental Research and Public Health, 10, 10: 4851-4868.

23. El-Fadel, M., Maroun, R., Quba'a, R. et al. (2014). «Determinants of diarrhea prevalence in urban slums: a comparative assessment towards enhanced environmental management», Environmental monitoring and assessment, 186, 2: 665-677.

24. Fink, G., Günther, I. \& Hill, K. (2013). «Urban Mortality Transitions: The Role of Slums», Program on the Global Demography of Aging PGDA Working Papers, 9913: 28.

25. Fink, G., Günther, I. \& Hill, K. (2014). «Slum Residence and Child Health in Developing Countries», Demography, DOI: 10.1007/s13524-014-0302-0.

26. Fnuap (2008). État de la population mondiale 2007. New York, UNFPA, $148 \mathrm{p}$.

27. Fournet, F., Meunier-Nikiema, A. \& Salem, G. (2013). Ouagadougou (1850-2004): une urbanisation différenciée (petit atlas urbain), IRD, $143 \mathrm{p}$.

28. Gossé, M. H. (1991). Villes et architectures: essai sur la dimension culturelle du développement, Karthala, 249 p. 
29. Graham, J. P. \& Polizzotto, M. L. (2013). «Pit latrines and their impacts on groundwater quality: a systematic review», Environmental Health Perspectives, 121, 5: 521-530.

30. Guengant, J. P., Lankoandé, M., Tapsoba, T. V. M. E. et al. (2009). Projections démographiques. Ouagoudougou, Institut National de la Statistique et de la Démographie, 108 p.

31. Guillaumot, L. (2005). Les moustiques et la dengue, Institut Pasteur de Nouvelle-Calédonie, $22 \mathrm{p}$.

32. Günther, I. \& Harttgen, K. (2012). «Deadly Cities? Spatial Inequalities in Mortality in sub-Saharan Africa», Population and Development Review, 38, 3: 469-486.

33. Hill, A., Hühner, T., Kreibich, V. et al. (2014). «Dar es Salaam, Megacity of Tomorrow: Informal Urban Expansion and the Provision of Technical Infrastructure», dans (dir.), Megacities. Springer: 165177.

34. Hingray, B. (1999). Comportement et modélisation hydrauliques des zones bâties en situation d'inondation. Géosciences. Montpellier, Université de Montpellier II-ORSTOM,244 p.

35. Hopewell, M. R. \& Graham, J. P. (2014). «Trends in access to water supply and sanitation in 31 major sub-Saharan African cities: an analysis of DHS data from 2000 to 2012», BMC Public Health 14, DOI: 10.1186/1471-2458-14-208.

36. INSD ET ICF International (2012). Enquête démographique et de santé et à indicateurs multiples du Burkina Faso 2010 (EDSBF-MICS IV), INSD et ORC Macro, $501 \mathrm{p}$.

37. Kafando, P., Segda, B. G., Nzihou, J. F. et al. (2013). «Environmental Impacts of Waste Management Deficiencies and Health Issues: A Case Study in the City of Kaya, Burkina Faso», Journal of Environmental Protection 2013, DOI: 10.4236/jep.2013.410124.

38. Kientga, S. (2008). Contribution du SIG à l'analyse des liens déchetssanté en milieu urbain dans les pays en développement. Cas de deux secteurs de la ville de Ouagadougou, Burkina Faso. Faculté Environnement Naturel, Architectural et Construit. Lausanne, École Polytechnique Fédérale de Lausanne, Thèse de doctorat, 213 p.

39. Kimani-Murage, E. W., Fotso, J. C., Egondi, T. et al. (2014). «Trends in childhood mortality in Kenya: The urban advantage has seemingly been wiped out», Health \& Place, 29, 1: 95-103.

40. Koné, B., Doumbia, M., SY, I. et al. (2014). «Étude des diarrhées en milieu périurbain à Abidjan par l'approche écosanté», VertigO-la revue électronique en sciences de l'environnement, DOI: 10.4000/vertigo. 14976 . 
41. Kouamé, P. K., Dongo, K., Nguyen-Viet, H. et al. (2014). «Ecohealth approach to urban waste management: Exposure to environmental pollutants and health risks in Yamoussoukro, Côte d'Ivoire», International Journal of Environmental Research and Public Health 11, DOI: 10.3390/ijerph111010292.

42. Mbaye, M., Mahé, G., Servat, E. et al. (2009). «Ressources en eau et santé publique au Sahel: exemple de la propagation des maladies infectieuses à Saint-Louis (Sénégal)», Science et Changements Planétaires/Sécheresse, 20, 1: 161-170.

43. Mondain, N., Arduin, P., Delaunay, V. et al. (2012). «La restitution des résultats dans les systèmes de surveillance démographique: une étude dans 3 sites africains», dans B. Schoumaker et D. Tabutin (dir.), Les systèmes d'information en démographie et en sciences sociales. Nouvelles questions, nouveaux outils?: Actes de la Chaire Quetelet 2006. Louvain-La-Neuve, Presses Universitaires de Louvain: 499-521.

44. Montgomery, M. R. \& Hewett, P. C. (2005). «Urban poverty and health in developing countries: Household and neighborhood effects», Demography, 42, 3: 397-425.

45. Mpakam, H. G., Kabeyne, K., Grelle, M. H. et al. (2006). «L'accès à l'eau potable et à l'assainissement dans les villes des pays en développement: cas de Basoussam (Cameroun)», VertigO-la revue électronique en sciences de l'environnement, DOI: 10.4000/vertigo. 2377.

46. Naing, C., Ren, W. Y., Man, C. Y. et al. (2011). «Awareness of dengue and practice of dengue control among the semi-urban community: A cross sectional survey», Journal of Community Health, 36, 6: 10441049.

47. Ngnikam, E., Mougoué, B., Feumba, R. et al. (2014). «L'eau, les déchets et la santé des enfants dans les quartiers à faible revenu de Yaoundé», dans (dir.), La Recherche Écosanté en pratique. Springer: 239-252.

48. OMS (2009). Normes de croissance OMS et identification de la malnutrition aiguë sévère chez l'enfance. Genève, OMS, $11 \mathrm{p}$.

49. ONU-Habitat (2010). L'état des villes africaines 2010. Gourvenance, inégalités et marchés fonciers urbains. Nairobi, ONU-Habitat, 266 p.

50. Piet Verdonschot, F. M. \& Besse-Lototskaya, A. A. (2014). «Flight distance of mosquitoes (Culicidae): A metadata analysis to support the management of barrier zones around rewetted and newly constructed wetlands», Limnologica-Ecology and Management of Inland Waters, 45: 69-79. 
51. Ponlawat, A., Scott, J. G. \& Harrington, L. C. (2005). «Insecticide susceptibility of Aedes aegypti and Aedes albopictus across Thailand», Journal of Medical Entomology, 42, 5: 821-825.

52. Rogier, C. (2003). «Paludisme de l'enfant en zone d'endémie: épidémiologie, acquisition d'une immunité et stratégies de lutte», Medecine Tropicale (Mars), 63, 4: 449-464.

53. Rossier, C., Soura, A., Baya, B. et al. (2012). «Profile: The Ouagadougou Health and Demographic Surveillance System», International Journal of Epidemiology, 41, 3: 658-666.

54. Rossier, C., Soura, A. \& Lankoande, B. (2013). «Migration et santé à la périphérie de Ouagadougou», Revue Quételet 1, 1: 91-118.

55. Rossier, C., Soura, A., Lankoandé, B. et al. (2011). Données collectées au Round 0, Round 1 et au Round 2: Rapport descriptif. Ouagadougou, ISSP, $71 \mathrm{p}$.

56. Sanga, B. (2011). Ouagadougou 2025. Des ambitions et des défis énormes. $6^{\mathrm{e}}$ Conférence africaine sur la population Infos $\mathrm{n}^{\mathrm{o}} 1,3 \mathrm{p}$.

57. Scott, L. M. \& Janikas, M. V. (2010). «Spatial statistics in ArcGIS», dans M. M. Fischer et A. Getis (dir.), Handbook of applied spatial analysis. Springer: 27-41.

58. Sheppard, Z. A., Norris, S. A., Pettifor, J. M. et al. (2009). «Approaches for assessing the role of household socioeconomic status on child anthropometric measures in urban South Africa», American Journal of Human Biology, 21, 1: 48-54.

59. Soura, A. B. (2009). Analyse de la mortalité et de la santé des enfants à Ouagadougou. Louvain, Presses universitaires de Louvain, 340 p.

60. Soura, A. B., Mberu, B., Elungata, P. et al. (2014). «Understanding Inequities in Child Vaccination Rates among the Urban Poor: Evidence from Nairobi and Ouagadougou Health and Demographic Surveillance Systems»,, Journal of Urban Health, DOI: 10.1007/s11524-014-9908-1.

61. Sy, I., Keita, M., Traoré, D. et al. (2014). «Eau, hygiène, assainissement et santé dans les quartiers précaires à Nouakchott (Mauritanie): contribution à l'approche écosanté à Hay Saken», VertigO-la revue électronique en sciences de l'environnement, DOI: 10.4000/vertigo.14999.

62. Sy, I., Tanner, M., Cissé, G. et al. (2011). «Vulnérabilité sanitaire et environnementale dans les quartiers défavorisés de Nouakchott (Mauritanie): analyse des conditions d'émergence et de développement de maladies en milieu urbain sahélien», VertigO-la revue électronique en sciences de l'environnement, DOI: 10.4000/vertigo.11174.

63. Un-Habitat (2014). The state of african cities 2014. Nairobi, UNHabitat, 267 p. 
64. UN. (2014). World Urbanization Prospects, 27 p.

65. Unger, A. (2013). «Children's health in slum settings», Archives of Disease in Childhood, DOI: 10.1136/archdischild-2011-301621

66. Vallée, J., Fournet, F., Meyer, P. E. et al. (2006). «Stratification de la ville de Ouagadougou (Burkina Faso) à partir d'une image panchromatique spot 5: une première étape à la mise en place d'une enquête de santé», Espace, Populations et Sociétés, 2, 3: 393-401.

67. Walker, C. L. F., Rudan, I., Liu, L. et al. (2013). «Global burden of childhood pneumonia and diarrhoea», The Lancet, 381, 9875: 14051416.

68. WHO \& UNICEF (2014). Progress on drinking water and sanitation, WHO/UNICEF, $78 \mathrm{p}$.

69. Yapi-Gnaoré, V. C., Vall, E., Havard, M. et al. (2014). Quatrième Semaine Scientifique Agricole de l'Afrique de l'Ouest et du Centre et 11 ème Assemblée Générale du CORAF/WECARD. Niamey, CORAF, $14 \mathrm{p}$. 